arbeitsrechtliche Maßnahmen bis hin zur außerordentlichen Kündigung des $\mathrm{Ar}$ beitsverhältnisses an. Da sich die Situation nicht verbesserte, kündigte der Chefarzt das Arbeitsverhältnis außerordentlich. Hiergegen klagte der Krankenhausträger. Im Juni 2011 kündigte der Krankenhausträger das Arbeitsverhältnis ordentlich zum 31.12.2011. Er war der Auffassung, dass die außerordentliche Kündigung des Chefarztes nicht berechtigt gewesen sei, da sie nicht aus wichtigem Grund ausgesprochen wurde. Er habe zum einen den Personalbedarf für die stationären und ambulanten Operationen nicht ordnungsgemäß und rechtzeitig angemeldet. Zum anderen sei ihm soweit möglich - OP-Personal zur Verfügung gestellt worden. Einen Anspruch auf eine bestimmte Personalausstattung habe er nicht. Der Chefarzt hielt dem entgegen, dass es ihm nicht zumutbar gewesen sei, das Arbeitsverhältnis bis zum 31.12.2011 aufrechtzuerhalten, nachdem ihn der Krankenhausträger trotz Abmahnungen nicht mit ausreichend nichtärztlichem Personal versorgt habe.

\section{Die Entscheidung}

Das Arbeitsgericht gab in erster Instanz zunächst dem Krankenhausträger Recht und hielt die außerordentliche Kündigung des Chefarztes für unwirksam. Hiergegen ging der Chefarzt in Berufung. Das Landesarbeitsgericht änderte das Urteil des Arbeitsgerichts Karlsruhe ab und wies die Klage nun ab. Die außerordentliche Kündigung des Chefarztes hat nach Auffassung des Gerichts das Arbeitsverhältnis fristlos beendet. Das Landesarbeitsgericht stellt fest, dass der Träger gegenüber dem Chefarzt wiederholt seine arbeitsvertragliche Pflicht, ihm das für seine Arbeit erforderliche nichtärztliche Personal zur Verfügung zu stellen, schuldhaft verletzt habe. Hierin sah das Gericht einen wichtigen Grund, um das Arbeitsverhältnis außerordentlich aufzulösen.

\section{Träger muss das notwendige Personal stellen}

Das Gericht sah in dem Arbeitsvertrag eine Verpflichtung des Trägers sozusagen als Gegenleistung für den Verzicht des Chefarztes auf seine Zulassung als Vertragsarzt, dass sich der Träger um die wirtschaftlichen Voraussetzungen des MVZ kümmert. Dazu gehöre ausdrücklich auch der Abschluss von Arbeitsverträgen mit nichtärztlichen Personen. Das Gericht ging davon aus, dass der Träger das MVZ so mit Personal auszustatten habe, wie es ein niedergelassener Arzt in Eigenregie tun würde, um seinen Praxisbetrieb einschließlich der ambulanten Operationen sicherzustellen. Schon die ausdrücklichen Regelungen des Arbeitsvertrages begründen nach Auffassung der Richter somit sowohl im Rahmen der arbeitsvertraglichen Aufgaben des Chefarztes als auch für die Nebentätigkeit die Verpflichtung des Trägers, dem Chefarzt das zu seiner Arbeit erforderliche nichtärztliche Personal zu überlassen. Dieselbe Verpflichtung, bezogen auf die arbeitsvertraglichen Aufgaben des Chefarztes, folge darüber hinaus aus der arbeitsvertraglichen Beschäftigungspflicht des Trägers. Der Träger konnte den Chefarzt nur bei entsprechender Personalausstattung vertragsgemäß als leitenden Arzt der plastisch-chirurgischen Abteilung beschäftigen. Hierzu bedurfte es sowohl einer Organisations- und Sprechstundenassistenz (Arzthelferin) als auch einer Operationsassistenz (bei stationären Operationen mindestens 3 Assistenzkräfte). Hiergegen habe der Träger mehrfach verstoßen. Dass das Nicht-zur-Verfügungstellen auf einer verspäteten OP-Anmeldung des Chefarztes beruhen sollte, sei in keinem Fall ersichtlich gewesen.

\section{Unzureichende Personalplanung}

Dem Träger wurde vom Gericht eine unzureichende Personalplanung attestiert. Eine Regelbesetzung mit 8,75 bzw. 9,75 Pflegekräften im OP-Dienst müsse bei 3 Operationssälen und mindestens einer Ambulanz (MVZ) in Krankheitsfällen oder bei Urlaub unweigerlich zu Personalengpässen führen. Das wiederholt vertragswidrige Vorenthalten von Personal kann nach Auffassung der Richter in einem Arztverhältnis wie dem vorliegenden grundsätzlich dessen außerordentliche Kündigung begründen, weil die Vertragsbeziehungen massiv gestört werden. Das Gericht würdigte insoweit, dass das Vorenthalten von Personal sich unter anderem auch unmittelbar auf die Vergütung des Arztes und auch seinen Ruf als zuverlässigen Arzt auswirken könne. Da der Chefarzt mehrfach den Sachverhalt abgemahnt hatte, sah es das Gericht nicht mehr als zumutbar an, das Arbeitsverhältnis weitere mehr als 8 Monate aufrechtzuerhalten. Er musste damit rechnen, dass der Träger das MVZ weiterhin nur unzureichend mit nichtärztlichem Personal versorgen werde.

\section{Fazit}

Dieser eher ungewöhnliche Fall einer eigenen außerordentlichen Kündigung eines Chefarztes wegen unzureichender Personaldecke macht deutlich, dass sich die kritische Personalsituation auch einmal sehr schnell gegen den Krankenhausträger wenden kann. Maßgeblich sind hierbei immer die einzelvertraglichen Regelungen im Arbeitsvertrag und die Interessenabwägung im Rahmen der Unzumutbarkeit der Fortsetzung des Arbeitsverhältnisses bei außerordentlicher Kündigung. Ärzten, die entsprechende Konstruktionen wie im vorliegenden Fall (Verzicht auf die Zulassung zugunsten einer Anstellung im MVZ) anstreben, ist zu raten, bei der Vertragsgestaltung insbesondere auch auf die notwendige vertragliche Verpflichtung zur Ausstattung mit dem notwendigen Personal zu achten. Wie das Urteil deutlich macht, kann der Anspruch auf ausreichend Personal aber unter Umständen bereits aus dem arbeitsrechtlichen Beschäftigungsanspruch - ohne vertragliche Regelung - hergeleitet werden.

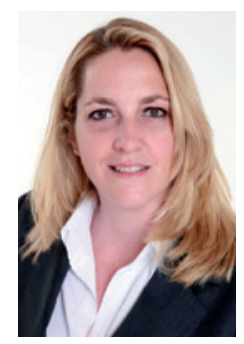

Korrespondenz Dr. iur. Isabel Häser Rechtsanwältin ECOVIS Lüdemann Wildfeuer \& Partner Sonnenstr. 9 80331 München www.ecovis.com 